J. Austral. Math. Soc. 20 (Series B) (1978), 401-433

\title{
THE CHARACTERISTIC IDENTITIES AND REDUCED MATRIX ELEMENTS OF THE UNITARY AND ORTHOGONAL GROUPS
}

\author{
M. D. GOULD
}

(Received 6 December 1977)

(Revised 29 September 1978)

\begin{abstract}
Polynomial identities satisfied by the generators of the Lie groups $O(n)$ and $U(n)$ are rederived. Using these identities the reduced matrix elements of the Lie groups $U(n)$ and $O(n)$ are evaluated as rational functions of the IR labels occurring in the canonical chains

$$
U(n) \supset U(n-1) \ldots \supset U(1) \text { and } O(n) \supset O(n-1) \ldots \supset O(2) \text {. }
$$

This method does not require an explicit realization of the Lie algebras and their representations using bosons. Finally, trace formulae encountered previously by several authors for finite dimensional irreducible representations are shown to hold on arbitrary representations admitting an infinitesimal character.
\end{abstract}

\section{Introduction}

The infinitesimal generators of semi-simple Lie groups are known $[5,6,11,13]$ to satisfy various polynomial identities in some specific irreducible representations. Identities of this form date back to the work of Lehrer-Ilamed [11] who showed that $n^{2}$ elements chosen from the enveloping algebra of any Lie algebra must satisfy $n^{2}$ identities which, in some cases, could be written in the form of a single polynomial identity of degree $n$ for an $n \times n$ matrix. Since the work of Ilamed such polynomial identities have been encountered by several authors $[5,6,13]$ for the Lie groups $G L(n)$ and its subgroups $S p(n)$ and $O(n)$.

The subject of the characteristic identities is closely related to the problem of obtaining a full set of invariants for a semi-simple Lie algebra. The full power of the characteristic identities was first recognized by Bracken and Green [5-7] who presented a systematic way of obtaining a full set of invariants for the Lie algebras 
of the general linear group and the orthogonal and symplectic subgroups. It was further shown how the eigenvalues of these invariants, on a given irreducible representation, may be expressed as a rational function of the IR labels. This work has since been generalized to arbitrary semi-simple Lie algebras $[2,4,9,17]$.

The methods of the characteristic identity have also been applied [8], in conjunction with the boson calculus, to the state labelling problems $O(n) \subset U(n)$ and $U(m) \subset S p(2 m)$.

In this paper we consider the characteristic identities satisfied by the generators of $O(n)$ and $U(n)$ and how they may be used to give some useful information concerning the subgroup embeddings $O(n) \subset O(n+1)$ and $U(n) \subset U(n+1)$.

We begin in Section 2 by assembling the generators of the Lie group $G L(n)$ into a matrix $a$ and then derive its characteristic identity. In Section 3 we show how we may obtain the eigenvalues, on an arbitrary maximal weight state, of the diagonal entries of any polynomial in the $G L(n)$ matrix $a$. We use these results to give an independent verification of Green's trace formula for arbitrary weights.

In Section 4 we look at the subgroup embedding $G L(n) \subset G L(n+1)$. In particular we consider certain $G L(n)$ vector and contragredient vector operators which may be constructed within the enveloping algebra of $G L(n+1)$. By contracting between the indices of a vector operator and a contragredient vector operator we obtain an interesting set of invariants. Invariants of this kind have already been applied to the state labelling problems [8] and in this section we obtain an explicit expression for these invariants in terms of the IR labels of $G L(n)$ and $G L(n+1)$. It is also noted that the $n \times n$ matrix of $G L(n)$ is embedded, in a natural canonical way, in the $(n+1) \times(n+1)$ matrix of $G L(n+1)$. The $(n+1, n+1)$ components of polynomials in the $G L(n+1)$ matrix are always $G L(n)$ invariants. We also show how these invariants may be expressed as a rational function of the IR labels of $G L(n)$ and those of $G L(n+1)$.

In Section 5 we apply some of our previous results to the evaluation of the reduced matrix elements of the generators of the unitary groups.

Finally, in Sections (6-8), we consider the extension of our previous results to the orthogonal subgroup of $G L(n)$. Unfortunately the orthogonal group requires an independent treatment due to the fact that the $O(n)$ generators with the metric $g_{i j}=\delta_{i j}$ are not in Cartan form. Hence an appropriate change of basis transformation has to be applied in order to proceed in analogy with $U(n)$.

\section{The $G L(n)$ characteristic identity}

Throughout this paper $F$ denotes a field of characteristic zero (which we usually take to be the real or complex field) and $g l(n, F)$ denotes the Lie algebra with basis

$$
\left\{a_{j}^{i} ; i, j=1, \ldots, n\right\}
$$


and Lie products

$$
\left[a_{j}^{l}, a_{l}^{k}\right]=\delta_{j}^{k} a_{l}^{l}-\delta_{l}^{i} a_{j}^{k} .
$$

The $g l(n, F)$ invariants $\sigma_{r}$, defined by (repeated affixes $i, j, k, \ldots$, are understood to be summed over from 1 to $n$ ) $\sigma_{1}=a_{i}^{i}, \sigma_{2}=a_{j}^{i} a_{i}^{j}, \sigma_{3}=a_{j}^{i} a_{k}^{j} a_{i}^{k}$ etc., form a full set of invariants for the Lie algebra. The centre of the enveloping algebra is therefore

$$
F\left[\sigma_{1}, \sigma_{2}, \ldots, \sigma_{n}\right]
$$

where $F\left[x_{1}, x_{2}, \ldots, x_{n}\right]$ denotes the polynomial algebra in indeterminates $x_{1}, \ldots, x_{n}$ over the underlying field $F$.

We choose as a Cartan subalgebra (C.S.A.) of $g l(n, F)$ the vector space spanned by the diagonal generators $a_{i}^{i}, i=1, \ldots, n$.

If $V$ is a finite dimensional irreducible representation of $g l(n, F)$ it is well known, from the second theorem of Cartan, that there exists a unique weight vector $v_{0}$ (up to scalar multiples) whose weight is greatest under the natural lexicographical ordering induced on $n$-tuples. Such a vector $v_{0}$ is defined by the conditions

$$
\begin{aligned}
& a_{i}^{i} v_{0}=\lambda_{i} v_{0}, \\
& a_{j}^{i} v_{0}=0 \text { for } j>i .
\end{aligned}
$$

We call $v_{0}$ a maximal weight vector of $g l(n, F)$ and we call its weight $\left(\lambda_{1}, \ldots, \lambda_{n}\right)$ the highest weight of $V$ (also referred to as the IR label of the representation).

The generators of $G L(n)$ may be assembled into a matrix $a$ whose $(i, j)$ entry is the generator $a_{j}^{i}$,

$$
a=\left(\begin{array}{cccc}
a_{1}^{1} & a_{2}^{1} & \ldots & a_{n}^{1} \\
a_{1}^{2} & a_{2}^{2} & \ldots & a_{n}^{2} \\
\vdots & & & \vdots \\
a_{1}^{n} & a_{2}^{n} & \ldots & a_{n}^{n}
\end{array}\right)
$$

We define powers of the matrix $a$ recursively by the formula

$$
\left(a^{m}\right)_{j}^{i}=a_{k}^{i}\left(a^{m-1}\right)_{i}^{k}=\left(a^{m-1}\right)_{k}^{i} a_{j}^{k} .
$$

In this way we may define polynomials in the matrix $a$. It can be shown by induction that if $p(x)$ is a polynomial over the field $F$ that we obtain the commutation relations

$$
\left[a_{j}^{i}, p(a)_{l}^{k}\right]=\delta_{j}^{k} p(a)_{l}^{i}-\delta_{l}^{i} p(a)_{j}^{k} .
$$

Green [5] discovered that, on an irreducible representation of $g l(n, F)$ with highest weight $\lambda$, the matrix $a$ satisfies the polynomial identity

$$
\prod_{r=1}^{n}\left(a-\lambda_{r}-n+r\right)=0
$$


In an effort to keep this paper as self-contained as possible we shall now present a simple proof of the identity (4).

Suppose $V$ is a finite dimensional irreducible representation of $g l(n, F)$ with highest weight $\lambda$ and highest weight vector $v_{0}$. It is convenient to introduce a set of polynomials

$$
p_{r}(x)=\prod_{k=r}^{n}\left(x-\lambda_{k}-n+k\right), \quad r=1, \ldots, n .
$$

By a simple recursion argument, using the commutation relations (3), we may verify the following result.

Proposition 1. Suppose $h(x)$ is a polynomial over the field $F$. Then

(i) $h(a)_{j}^{i} v_{0}=0$ for $i<j$.

(ii) $h(a)_{i}^{i} v_{0}=\alpha_{i} v_{0}, \alpha_{i} \in F$.

(iii) If $p_{r}(x)$ divides $h(x)$ then $h(a)_{j}^{i} v_{0}=0$ for $i, j \geqslant r$.

As a result of part (iii) of this proposition we have $p_{1}(a)_{j}^{i} v_{0}=0$ for $i, j=1, \ldots, n$ and the identity (4) holds on the highest weight vector $v_{0}$. From this it is an easy matter to prove that the identity is satisfied on all of $V$. To this end let us denote by $\operatorname{Ann} p_{1}(x)$ the set

$$
\left\{v \in V \text { such that } p_{1}(a)_{j}^{i} v=0 ; i, j=1, \ldots, n\right\} .
$$

Clearly Ann $p_{1}(x)$ is a sub-representation of $V$. Thus, by irreducibility, Ann $p_{1}(x)$ is either zero or all of $V$. Since $v_{0} \in \operatorname{Ann} p_{1}(x)$, Ann $p_{1}(x)$ must be all of $V$ and the identity (4) is proved.

We note here that the proof just presented holds for arbitrary standard cyclic representations (that is representations generated by a maximal weight vector) and in particular infinite dimensional irreducible representations possessing a maximal weight vector.

Following Green [5] we may define an adjoint $\bar{a}$ of the matrix $a$ by setting

$$
\bar{a}_{j}^{i}=-a_{j}^{i}
$$

We then define powers of the matrix $\bar{a}$ by the formula

$$
\left(\bar{a}^{m}\right)_{i}^{j}=\left(\bar{a}^{m-1}\right)_{i}^{k} \bar{a}_{k}^{j}=\bar{a}_{i}^{k}\left(\bar{a}^{m-1}\right)_{k}^{j}
$$

In this case we consider a set of polynomials

$$
q_{r}(x)=\prod_{k=1}^{r}\left(x+\lambda_{r}-r+1\right), \quad r=1, \ldots, n
$$

and we may verify the following result. 
Proposirion 2. Let $v_{0}$ be a maximal weight vector of $g l(n, F)$ with weight $\lambda$ and suppose $p(x)$ is a polynomial over $F$. Then

(i) $p(\bar{a})_{i}^{j} v_{0}=0$ for $j<i$.

(ii) $p(\bar{a})_{i}^{i} v_{0}=\beta_{i} v_{0}, \beta_{i} \in F$.

(iii) If $q_{r}(x)$ divides $p(x)$ then $p(\bar{a})_{j}^{i} v_{0}=0$ for $i, j \leqslant r$.

From this result we may prove that the identity

$$
\prod_{r=1}^{n}\left(\tilde{a}+\lambda_{r}-r+1\right)=0
$$

is satisfied on an irreducible representation with highest weight $\lambda=\left(\lambda_{1}, \ldots, \lambda_{n}\right)$. We call equation (5) the $g l(n, F)$ adjoint identity.

It is important to note that identities of the types (4) and (5) are not restricted to representations generated by a maximal weight vector. Recent work by O'Brien, Carey and Cant [16] shows that the matrix $a$ satisfies a polynomial identity $p(a)=0$ where $p(x)$ is a monic polynomial of degree $n$ whose coefficients lie in the centre of the enveloping algebra. From this work we see that identities of the types (4) and (5) hold on any representation of $g l(n, F)$ which admits an infinitesimal character $\chi$ (that is any representation on which the fundamental invariants $\sigma_{r}$ take constant values $\chi\left(\sigma_{r}\right) \in F$ ). From a well-known theorem of Harish Chandra [10] we know that any character $\chi$ is equal to the character $\chi_{\lambda}$ of an irreducible representation $V(\lambda)$ of $g l(n, F)$ with highest weight $\lambda$. On any such representation the identities (4) and (5) hold.

Throughout the remainder of this paper we shall write the characteristic identity in the form

$$
\prod_{r=1}^{n}\left(a-\alpha_{r}\right)=0
$$

where the operators $\alpha_{r}$ are invariants of the Lie algebra whose eigenvalues on a representation admitting an infinitesimal character $\chi_{\lambda}$ are given by

$$
\alpha_{r}=\lambda_{r}+n-r
$$

The operators $\alpha_{r}$ lie in an algebraic extension of the enveloping algebra of $g l(n, F)$ and are related to the fundamental invariants $\sigma_{r}$ by equations of the form

$$
\sigma_{1}=\sum_{r=1}^{n}\left(\alpha_{r}-n+r\right), \quad \sigma_{2}=\sum_{r=1}^{n}\left(\alpha_{r}-n+r\right)\left(\alpha_{r}+1-r\right) \ldots
$$

(See Green [7] for a more general expression.)

From now on we refer to the operators $\alpha_{r}$ as the characteristic roots of $g l(n, F)$. 
From the characteristic identities satisfied by the matrices $a$ and $\bar{a}$ we may construct a set of projection operators

$$
\begin{aligned}
& f_{r}=\prod_{k=1, k \neq r}^{n}\left(\frac{a-\alpha_{k}}{\alpha_{r}-\alpha_{k}}\right), \\
& \tilde{f}_{r}=\prod_{k=1, k \neq r}^{n}\left(\frac{\bar{a}+\alpha_{k}-n+1}{\alpha_{k}-\alpha_{r}}\right),
\end{aligned}
$$

which satisfy

$$
\sum_{r=1}^{n}\left(f_{r}\right)_{j}^{i}=\delta_{j}^{i}, \quad \sum_{r=1}^{n}\left(\bar{f}_{r}\right)_{j}^{i}=\delta_{j}^{i}
$$

and the orthogonality conditions

$$
f_{r} f_{k}=\delta_{r k} f_{k}, \quad \tilde{f}_{r} \tilde{f}_{k}=\delta_{r k} \tilde{f}_{k} .
$$

More generally, if $p(x)$ is any polynomial over $F$, we may write (see Green [7])

$$
\begin{aligned}
& p(a)=\sum_{r=1}^{n} p\left(\alpha_{r}\right) f_{r}, \\
& p(\bar{a})=\sum_{r=1}^{n} p\left(n-1-\alpha_{r}\right) \bar{f}_{r} .
\end{aligned}
$$

By means of this formula rather general functions of the matrix $a$ may be defined. In particular we may define an inverse of the matrix $p(a)$ by setting

$$
p^{-1}(a)=\sum_{r=1}^{n} p\left(\alpha_{r}\right)^{-1} f_{r} .
$$

From the characteristic identity it is easily checked that the matrix $p^{-1}(a)$ satisfies the usual properties of an inverse matrix,

$$
p(a)_{j}^{i} p^{-1}(a)_{k}^{j}=p^{-1}(a)_{j}^{i} p(a)_{k}^{j}=\delta_{k}^{i} .
$$

\section{Casimir invariants}

From the commutation relations (3) we see that traces of arbitrary polynomials in the matrix $a$, in particular the

$$
\sigma_{r}=\operatorname{tr}\left(a^{r}\right)
$$

are invariants of the Lie algebra.

It is our aim in this section to evaluate the eigenvalues of such invariants when acting on representations admitting a character $\chi_{\lambda}$. 
From equations (9) we may write

$$
\begin{aligned}
& \operatorname{tr} p(a)=\sum_{k=1}^{n} p\left(\alpha_{k}\right) \operatorname{tr} f_{k}, \\
& \operatorname{tr} p(\bar{a})=\sum_{k=1}^{n} p\left(n-1-\alpha_{k}\right) \operatorname{tr} \tilde{f}_{k} .
\end{aligned}
$$

It therefore suffices to evaluate the eigenvalues of the invariants $\operatorname{tr} f_{k}$ and $\operatorname{tr} f_{k}$.

Recently Green [7] (see also Edwards [2]) has obtained a formula relating the eigenvalues of these invariants, when acting on a finite dimensional irreducible representation, to the components of the highest weight. However, the method employed by Green relies heavily on the finite dimensionality and irreducibility of the representations concerned. We present here a more general approach which shows that Green's trace formula extends to arbitrary representations admitting an infinitesimal character.

We shall in fact prove more than what is needed here. From Propositions 1 and 2, part (ii) we see that if $p(x)$ is a polynomial then the operators $p(a)_{i}^{i}$ and $p(\bar{a})_{i}^{i}$ take constant values on a maximal weight vector. We shall determine the eigenvalues of these operators when acting on arbitrary maximal weight states of $g l(n, F)$. In view of equation (9) this may be done if we can evaluate the eigenvalues of the $\left(f_{k}\right)_{r}^{r}$ and $\left(\bar{f}_{k}\right)_{r}^{r}$ when acting on a maximal weight vector.

Throughout the remainder of this section we let $v_{0}$ denote a maximal weight vector of arbitrary weight $\mu=\left(\mu_{1}, \ldots, \mu_{n}\right)$.

We begin by introducing a set of operators

$$
\begin{gathered}
p_{i}(k)=\prod_{j=i}^{n}\left(a-\alpha_{j}\right)_{k}^{k}, \\
p_{i, j}(k)=\prod_{l=i l \neq \neq j}^{n}\left(a-\alpha_{l}\right)_{k}^{k} .
\end{gathered}
$$

From definition it is clear that $p_{i, j}(k)=p_{i}(k)$ if $j<i$.

Now let $\rho_{i, j}(k)$ and $\rho_{i}(k)$ denote the eigenvalues of the operators $p_{i, j}(k)$ and $p_{i}(k)$ respectively when acting on the maximal weight vector $v_{0}$. Then using Proposition 1, part (i) together with the commutation relations (3), it is easily verified that the $\rho_{i}(k)$ satisfy the difference equation

$$
\rho_{i}(k)=\left(\alpha_{k}-\alpha_{i}\right) \rho_{i+1}(k)-\rho_{i+1}(k+1)-\ldots-p_{i+1}(n),
$$

where the roots $\alpha_{r}$ take constant values $\mu_{r}+n-r$. Similarly when $i<j-1$ the $\rho_{i, j}(k)$ satisfy the difference equation

$$
\rho_{i, j}(k)=\left(\alpha_{k}-\alpha_{i}\right) \rho_{i+1, j}(k)-\rho_{i+1, j}(k+1)-\ldots-\rho_{i+1, j}(n)
$$


and when $i=j-1$ we obtain

$$
\rho_{j-1, j}(k)=\left(\alpha_{k}-\alpha_{j-1}\right) \rho_{j+1}(k)-\rho_{j+1}(k+1)-\ldots \rho_{j+1}(n) .
$$

From Proposition 1, part (iii) it is clear that $\rho_{i}(k)=0$ for $k \geqslant i$. Similarly when $i \leqslant j$ we have $\rho_{i, j}(k)=0$ for $k>j$. This set of conditions together with the extra boundary condition

$$
\rho_{i, n}(n)=\prod_{l=i}^{n-1}\left(\alpha_{n}-\alpha_{l}\right)
$$

enables us to solve these difference equations uniquely.

In particular the $\rho_{1, r}(k)$ are easily computed for $k \leqslant r$ and we obtain the formula

$$
\rho_{1, r}(k)=\prod_{l<k}\left(\alpha_{r}-\alpha_{l}\right) \prod_{D k}\left(\alpha_{r}-\alpha_{l}-1\right), \quad k \leqslant r .
$$

Now from definition the eigenvalues of the operators $\left(f_{r}\right)_{k}^{k}$ are related to the $\rho_{l, r}(k)$ by the formula

$$
\left(f_{r}\right)_{k}^{k} v_{0}=\prod_{l \neq r}\left(\alpha_{r}-\alpha_{l}\right)^{-1} \rho_{l, r}(k) v_{0}
$$

Substituting formula (12) into this expression yields the formula

$$
\left(f_{r}\right)_{k}^{k} v_{0}=\prod_{l>k}\left(\frac{\alpha_{r}-\alpha_{l}-1}{\alpha_{r}-\alpha_{l}+\delta_{r l}}\right) v_{0}, \quad k \leqslant r .
$$

Of course, when $k>r$, we have, from Proposition 1,

$$
\left(f_{r}\right)_{k}^{k} v_{0}=0
$$

By a similar procedure we may also verify the result

$$
\left(\bar{f}_{r}\right)_{k}^{k} v_{0}=\left\{\begin{array}{l}
\prod_{l<k}\left(\frac{\bar{\alpha}_{r}-\bar{\alpha}_{l}-1}{\bar{\alpha}_{r}-\bar{\alpha}_{l}+\delta_{r l}}\right) v_{0}, \quad k \geqslant r, \\
0, \quad k<r .
\end{array}\right.
$$

By summing equations (13) and (14) over $k$ we obtain the result

$$
\begin{aligned}
& \chi_{i \mu}\left(\operatorname{tr} f_{k}\right)=\prod_{l \neq k}\left(\frac{\alpha_{k}-\alpha_{l}-1}{\alpha_{k}-\alpha_{l}}\right), \\
& \chi_{\mu}\left(\operatorname{tr} \tilde{f}_{k}\right)=\prod_{l \neq k}\left(\frac{\alpha_{k}-\alpha_{l}+1}{\alpha_{k}-\alpha_{l}}\right) \quad\left(\alpha_{k}=\mu_{k}+n-k\right),
\end{aligned}
$$

which is the required generalization of Green's formula to arbitrary weights $\mu$. 


\section{Spectral resolution}

In this section we apply the characteristic identities of the Lie groups $G L(n)$ and $G L(n+1)$ to give some useful information concerning the subgroup embedding $G L(n) \subset G L(n+1)$. We first require some preliminary remarks concerning vector operators.

We define a $g l(n, F)$ vector operator as a collection of $n$ operators $\psi^{i}$ which satisfy the conditions

$$
\left[a_{j}^{i}, \psi^{k}\right]=\delta_{j}^{k} \psi^{i} .
$$

We denote such a vector operator by $\psi$ and we refer to the operators $\psi^{i}$ as the components of $\psi$.

Similarly, we define a $g l(n, F)$ contragredient vector operator $\phi$ as an operator with $n$ components $\phi_{i}$ which satisfy

$$
\left[a_{j}^{i}, \phi_{k}\right]=-\delta_{k}^{i} \phi_{j} .
$$

It is known [5] that a vector operator $\psi$ may be resolved into a sum of vector operators $\psi[r]$,

$$
\psi=\sum_{r=1}^{n} \psi[r]
$$

where each $\psi[r]$ is a vector operator which increases the eigenvalue of the label $\lambda_{r}$ by one unit leaving the other $\lambda_{k}$ unchanged, that is,

$$
\lambda_{k} \psi[r]=\psi[r]\left(\lambda_{k}+\delta_{k r}\right)
$$

Hence, in terms of our roots $\alpha_{k}$, we have

$$
\alpha_{k} \psi[r]=\psi[r]\left(\alpha_{k}+\delta_{k r}\right)
$$

Similarly a contragredient vector operator $\phi$ may be resolved into a sum of

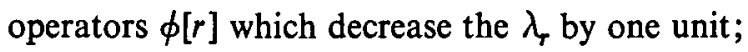

$$
\alpha_{k} \phi[r]=\phi[r]\left(\alpha_{k}-\delta_{k r}\right)
$$

Since each component $\psi[r]$ (respectively $\phi[r]$ ) is a vector (respectively contragredient vector) operator it is clear that any linear combination of such operators is also a vector (respectively contragredient vector) operator.

Following Green $[5,7]$ the $\psi[r]$ and $\phi[r]$ may be constructed by application of the projection operators $f_{r}$ and $\tilde{f}_{r}$,

$$
\begin{aligned}
& \psi[r]=f_{r} \psi=\psi f_{r}, \\
& \phi[r]=f_{r} \phi=\phi f_{r} .
\end{aligned}
$$


From the characteristic identities it then follows that, if $p(x)$ is any polynomial, then

$$
p(a) \psi[r]=p\left(\alpha_{r}\right) \psi[r]
$$

In particular we have

$$
f_{k} \psi[r]=\delta_{k r} \psi[r] .
$$

Finally, we note that this orthogonality relation implies that the vector operators $\psi[r]$ form a linearly independent set. For suppose

$$
\sum_{r=1}^{n} \gamma_{r} \psi[r]=0,
$$

where the $\gamma_{r}$ are invariant multiples of the identity matrix $a^{0}$. Then applying the projection $f_{k}$ to the left of this equation gives

$$
\gamma_{k} \psi[k]=0,
$$

which in turn implies each $\gamma_{k}$ is zero.

We now consider $g l(n+1, F)$ and its associated characteristic identity.

We denote the $g l(n+1, F)$ matrix by $b$, that is

$$
b=\left(\begin{array}{cccc|c}
a_{1}^{1} & a_{2}^{1} & \ldots & a_{n}^{1} & a_{n+1}^{1} \\
a_{1}^{2} & a_{2}^{2} & \ldots & a_{n}^{2} & a_{n+1}^{2} \\
\vdots & \vdots & \ldots & \vdots & \vdots \\
a_{1}^{n} & a_{2}^{n} & \ldots & a_{n}^{n} & a_{n+1}^{n} \\
\hdashline a_{1}^{n+1} & a_{2}^{n+1} & \ldots & a_{n}^{n+1} & a_{n}^{n+1}
\end{array}\right) .
$$

We note that the first $n$ rows and columns of the matrix $b$ give us the matrix $a$ considered earlier. The first $n$ entries of the last column constitute a $g l(n, F)$ vector operator while the first $n$ entries of the last row constitute a contragredient vector operator. The remaining entry $a_{n+1}^{n+1}$ is a $g l(n, F)$ invariant.

Using the commutation relations (3) for $g l(n+1, F)$ we may verify that if $p(x)$ is any polynomial over the underlying field $F$ then the first $n$ entries of the last column of the matrix $p(b)$ (the $p(b)_{n+1}^{i}$ ) constitute a $g l(n, F)$ vector operator while the $p(b)_{i}^{n+1}$ constitute a contragredient vector operator. The $(n+1, n+1)$ entry $p(b)_{n+1}^{n+1}$ is always a $g l(n, F)$ invariant and it is one of our aims in this section to determine these invariants as rational functions of the roots appearing in the $g l(n, F)$ and $g l(n+1, F)$ identities.

We write the $g l(n+1, F)$ characteristic identity in the form

$$
\prod_{r=1}^{n+1}\left(b-\beta_{r}\right)=0,
$$


where the operators $\beta_{r}$ are invariants of the Lie algebra which take constant values $\lambda_{r}+n+1-r$ when acting on an irreducible representation with highest weight $\left(\lambda_{1}, \lambda_{2}, \ldots, \lambda_{n+1}\right)$. They are related to the fundamental invariants $\widehat{\sigma}_{k}=\operatorname{tr}\left(b^{k}\right)$ by equations analogous to the equations (6) for $g l(n, F)$. Since the $g l(n+1, F)$ roots $\beta_{r}$ are $g l(n+1, F)$ invariants they are also invariants of $g l(n, F)$ and we must have $\left[\beta_{r}, \alpha_{k}\right]=0$. Although the $g l(n, F)$ roots $\alpha_{r}$ are $g l(n, F)$ invariants they are not invariants of $g l(n+1, F)$. However, in our previous notation, the $a_{n+1}^{i}$ and the $a_{i}^{n+1}$ may be resolved into shift vectors

$$
a_{n+1}^{i}=\sum_{r=1}^{n} \psi[r]^{i}, \quad a_{i}^{n+1}=\sum_{r=1}^{n} \phi[r]_{i}, \quad i=1, \ldots, n .
$$

From equations (17) and (18) we readily obtain

$$
\left[\alpha_{r}, a_{n+1}^{i}\right]=\psi[r]^{i} \text { while }\left[\alpha_{r}, a_{i}^{n+1}\right]=-\phi[r]_{i} .
$$

Finally, we have

$$
\left[\alpha_{r}, a_{n+1}^{n+1}\right]=0 .
$$

As for $g l(n, F)$ we may construct the $g l(n+1, F)$ projection operators

$$
\begin{aligned}
& g_{k}=\prod_{l=1, l \neq k}^{n+1}\left(\frac{b-\beta_{l}}{\beta_{k}-\beta_{l}}\right), \\
& \bar{g}_{k}=\prod_{l=1, l \neq k}^{n+1}\left(\frac{b+\beta_{l}-n}{\beta_{l}-\beta_{k}}\right),
\end{aligned}
$$

which satisfy the orthogonality conditions

$$
g_{k} g_{r}=\delta_{k r} g_{r}, \quad \bar{g}_{k} \bar{g}_{r}=\delta_{k r} \bar{g}_{r} .
$$

Furthermore, if $p(x)$ is any polynomial over the underlying field, we may write

$$
\begin{aligned}
& p(b)=\sum_{k=1}^{n+1} p\left(\beta_{k}\right) g_{k}, \\
& p(\bar{b})=\sum_{k=1}^{n+1} p\left(n-\beta_{k}\right) \bar{g}_{k} .
\end{aligned}
$$

From the $g l(n+1, F)$ identity we have

$$
b g_{k}=\beta_{k} g_{k} .
$$

Taking the $(i, n+1)$ entry of this matrix equation we have

$$
\sum_{l=1}^{n+1} a_{l}^{i}\left(g_{k}\right)_{n+1}^{l}=\beta_{k}\left(g_{k}\right)_{n+1}^{l} .
$$

Rearranging this expression, we obtain

$$
a_{n+1}^{i}\left(g_{k}\right)_{n+1}^{n+1}=\left(\beta_{k}-a\right)_{j}^{i}\left(g_{k}\right)_{n+1}^{j}, \quad i=1, \ldots, n .
$$


Similarly, we may write

$$
\left(g_{k}\right)_{n+1}^{n+1} a_{i}^{n+1}=\left(g_{k}\right)_{j}^{n+1}\left(\beta_{k}-a\right)_{i}^{j}, \quad i=1, \ldots, n .
$$

For simplicity we shall denote the $g l(n, F)$ invariant $\left(g_{k}\right)_{n+1}^{n+1}$ by $\varepsilon_{k}$. From equation (21) we have

$$
p(b)_{n+1}^{n+1}=\sum_{k=1}^{n+1} p\left(\beta_{k}\right) \varepsilon_{k}
$$

So in order to evaluate the invariants $p(b)_{n+1}^{n+1}$ as a rational function of the $\beta_{k}$ and $\alpha_{r}$ it suffices to evaluate the $\varepsilon_{k}$.

From equation (10) we may invert (22) and (23) by writing

$$
\begin{aligned}
& \left(g_{k}\right)_{n+1}^{i}=\sum_{r=1}^{n} \psi[r]^{i}\left(\beta_{k}-\alpha_{r}-1\right)^{-1} \varepsilon_{k}, \\
& \left(g_{k}\right)_{i}^{n+1}=\sum_{r=1}^{n} \varepsilon_{k}\left(\beta_{k}-\alpha_{r}-1\right)^{-1} \phi[r]_{i},
\end{aligned}
$$

where we have used

and

$$
\left(\beta_{k}-\alpha_{r}\right)^{-1} \psi[r]=\psi[r]\left(\beta_{k}-\alpha_{r}-1\right)^{-1}
$$

$$
\left(\beta_{k}-\alpha_{r}-1\right)^{-1} \phi[r]=\phi[r]\left(\beta_{k}-\alpha_{r}\right)^{-1} .
$$

However, from equation (21), we have

$$
\sum_{k=1}^{n+1}\left(g_{k}\right)_{n+1}^{i}=\delta_{n+1}^{i}=0 \text { for } i=1, \ldots, n .
$$

Hence summing equation (25) over $k$ from 1 to $n+1$ we obtain

$$
\sum_{r=1}^{n} \psi[r]\left(\sum_{k=1}^{n+1}\left(\beta_{k}-\alpha_{r}-1\right)^{-1} \varepsilon_{k}\right)=0 .
$$

From the independence of the $\psi[r]$ this immediately implies that

$$
\sum_{k=1}^{n+1}\left(\beta_{k}-\alpha_{r}-1\right)^{-1} \varepsilon_{k}=0, \quad r=1, \ldots, n .
$$

This set of equations together with the condition

$$
\sum_{k=1}^{n+1} \varepsilon_{k}=1\left(\sum_{k=1}^{n+1}\left(g_{k}\right)_{n+1}^{n+1}=\delta_{n+1}^{n+1}\right)
$$

uniquely determines the $\varepsilon_{k}$. These equations are readily solved (using matrix methods for example) and yield the solution

$$
\varepsilon_{k}=\prod_{p=1, p \neq k}^{n+1}\left(\beta_{k}-\beta_{p}\right)^{-1} \prod_{l=1}^{n}\left(\beta_{k}-\alpha_{l}-1\right) .
$$


Substituting this expression into equation (24) allows us to evaluate invariants of the form $p(b)_{n+1}^{n+1}$ for any polynomial $p(x)$.

The remainder of this section is devoted to the evaluation of the invariants $\phi[r]_{i} \psi[r]^{i}$ and $\psi[r]^{i} \phi[r]_{i}$. We begin by inverting equation (25) to get $\psi[r]^{i}$ in the form $p(b)_{n+1}^{i}$ for a suitably defined polynomial $p(x)$. We may then evaluate our invariants by a simple application of the $g l(n+1, F)$ characteristic identity.

Let us first consider the solutions $\gamma_{l k}$ to the equations

$$
\sum_{k=1}^{n+1} \gamma_{l k} \varepsilon_{k}\left(\beta_{k}-\alpha_{r}-1\right)^{-1}=\delta_{r l}, \quad l=1, \ldots, n,
$$

and

$$
\sum_{k=1}^{n+1} \gamma_{l k} \varepsilon_{k}=0
$$

Then, for each $r=1, \ldots, n$, we have $(n+1)$ equations in $(n+1)$ unknowns $\gamma_{r k}(k=1, \ldots, n+1)$. Again these equations may be solved and yield the solution

where

$$
\gamma_{r k}=\gamma_{r}\left(\beta_{k}-\alpha_{r}-1\right)^{-1}
$$

$$
\gamma_{r}=(-1)^{n} \prod_{p=1}^{n+1}\left(\beta_{p}-\alpha_{r}-1\right) \prod_{l=1, l+r}^{n}\left(\alpha_{r}-\alpha_{l}\right)^{-1} .
$$

Now from equation (25) we may write

$$
\sum_{k=1}^{n+1}\left(g_{k}\right)_{n+1}^{i} \gamma_{r k}=\sum_{l=1}^{n} \psi[l]^{i} \sum_{k=1}^{n+1} \gamma_{r k} \varepsilon_{k}\left(\beta_{k}-\alpha_{l}-1\right)^{-1}
$$

Rearranging this expression using equation (29) we readily obtain

$$
\psi[r]^{i}=\sum_{k=1}^{n+1}\left(g_{k}\right)_{n+1}^{i} \gamma_{r}\left(\beta_{k}-\alpha_{r}-1\right)^{-1} .
$$

The invariants $\gamma_{r}$ appearing in this equation have an interesting interpretation. From the orthogonality of the projections $f_{r}$ and equation (19) we have

$$
\phi[r]_{i} \psi[r]^{i}=a_{i}^{n+1} \psi[r]^{i}
$$

Hence, multiplying equation (31) by $a_{i}^{n+1}$ and summing over $i$ from 1 to $n$ we obtain

$$
\phi[r]_{2} \psi[r]^{i}=\gamma_{r}
$$

where we have used the result

$$
\sum_{k=1}^{n+1} a_{i}^{n+1}\left(g_{k}\right)_{n+1}^{i}\left(\beta_{k}-\alpha_{>}-1\right)^{-1}=\sum_{k=1}^{n+1} \varepsilon_{k}=1,
$$

which is verifiable with the help of the $g l(n+1, F)$ characteristic identity and equation (29). 
In a similar way, by considering the operators $\left(\tilde{g}_{k}\right)_{n+1}^{i}$ and $\left(\tilde{g}_{k}\right)_{i}^{n+1}$, we may also verify that

$$
\left(\bar{g}_{k}\right)_{n+1}^{i}=\sum_{r=1}^{n} \bar{\varepsilon}_{k}\left(\beta_{k}-\alpha_{r}\right)^{-1} \psi[r]^{i},
$$

where

$$
\bar{\varepsilon}_{k}=\left(\bar{g}_{k}\right)_{n+1}^{n+1}=\prod_{p=1, p \neq k}^{n+1}\left(\beta_{k}-\beta_{p}\right)^{-1} \prod_{l=1}^{n}\left(\beta_{k}-\alpha_{t}\right)
$$

We may then invert (33) by writing

$$
\psi[r]^{i}=\sum_{k=1}^{n+1} \bar{\nu}_{r}\left(\beta_{k}-\alpha_{,}\right)^{-1}\left(\bar{g}_{4}\right)_{n+1}^{i} .
$$

Using this result and the orthogonality of the projections $\hat{f}_{r}$ we may then verify that

where

$$
\psi[r]^{i} \phi[r]_{i}=\bar{\gamma}_{r}
$$

$$
\bar{\gamma}_{r}=(-1)^{n} \prod_{p=1}^{n+1}\left(\beta_{p}-\alpha_{r}\right) \prod_{l=1, \downarrow \neq r}^{n}\left(\alpha_{r}-\alpha_{\iota}\right)^{-1} .
$$

Finally, we consider the operators $\psi[r] \gamma_{r}^{-1} \phi[r]$. From equation (31), we have

$$
\psi[r]^{i} \gamma_{r}^{-1} \phi[r]_{j}=\sum_{k=1}^{n+1}\left(g_{k}\right)_{n+1}^{i}\left(\beta_{k}-\alpha_{r}-1\right)^{-1} \phi[r]_{j} .
$$

Summing this equation over $r$ using equation (26) we may write

$$
\sum_{r=1}^{n} \psi[r]^{i} \gamma_{r}^{-1} \phi[r]_{j}=\sum_{k=1}^{n+1}\left(g_{k}\right)_{n+1}^{i} \varepsilon_{k}^{-1}\left(g_{k}\right)_{j}^{n+1}
$$

However, from the work of Green [7] (see the Appendix) we have

$$
\sum_{k=1}^{n+1}\left(g_{k}\right)_{n+1}^{i} \varepsilon_{k}^{-1}\left(g_{k}\right)_{j}^{n+1}=\delta_{j}^{i}
$$

From this result and the orthogonality relation (20) we obtain

Similarly we show that

$$
\psi[r]^{i} \gamma_{r}^{-1} \phi[r]_{j}=\left(f_{r}\right)_{j}^{i}
$$

$$
\phi[r]_{j} \bar{\gamma}_{r}^{-1} \psi[r]^{i}=\left(\tilde{f}_{r}\right)_{j^{*}}^{i}
$$

Hence if $p(x)$ is a polynomial over the field $F$ we may write

$$
\begin{aligned}
& p(a)=\sum_{r=1}^{n} p\left(\alpha_{r}\right) \psi[r] \gamma_{r}^{-1} \phi[r], \\
& p(\bar{a})=\sum_{r=1}^{n} p\left(n-1-\alpha_{r}\right) \phi[r] \bar{\gamma}_{r}^{-1} \psi[r] .
\end{aligned}
$$


Such resolutions are useful and may be regarded as a generalization of the spectral resolution of a non-degenerate numerical matrix. Of particular interest are the invariants $\gamma_{r}$ and $\bar{\gamma}_{r}$ because they are closely related to the reduced matrix elements of the generators $a_{n+1}^{i}$ and $a_{i}^{n+1}$.

\section{Reduced matrix elements of $U(n)$}

The generators $a_{j}^{i}$ of the Lie group $U(n)$ satisfy the commutation relations

$$
\left[a_{j}^{i}, a_{l}^{k}\right]=\delta_{j}^{k} a_{l}^{i}-\delta_{l}^{i} a_{j}^{k}
$$

and moreover satisfy the Hermiticity property

$$
\left(a_{j}^{i}\right)^{\dagger}=a_{i}^{j} .
$$

All of our previous results obtained for $G L(n)$ go through unchanged to $U(n)$ [7].

From equation (38) we see that the $U(n)$ invariants $\sigma_{r}$ are Hermitian operators so we deduce that the $U(n)$ roots $\alpha_{r}$ may likewise be regarded as Hermitian.

By a simple induction argument we may readily verify that if $p(x)$ is any polynomial over the underlying field $F$ then

$$
p(a)_{j}^{i \dagger}=p(a)_{i}^{j} \text {. }
$$

Suppose now we look at the subgroup embedding $U(n) \subset U(n+1)$. Following our previous notation let $\psi[r]^{i}$ and $\phi[r]_{i}$ be the operators defined by

$$
\begin{aligned}
& \psi[r]^{i}=\left(f_{r}\right)_{j}^{i} a_{n+1}^{j}=a_{n+1}^{j}\left(f_{r}^{r}\right)_{j}^{i}, \\
& \phi[r]_{i}=a_{j}^{n+1}\left(f_{r}\right)_{j}^{i}=\left(f_{r}\right)_{j}^{i} a_{j}^{n+1} .
\end{aligned}
$$

We then have

$$
\psi^{\dagger}[r]=\phi[r]
$$

So from now on we write $\psi^{\dagger}[r]$ in place of $\phi[r]$.

We now note that the generators $a_{j}^{i}$ where $i$ and $j$ are restricted to values $1, \ldots, m$ (for some positive integer $m$ less than $n$ ) form the generators of the unitary subgroup $U(m)$ of $U(n)$. We see therefore that $U(n)$ admits the canonical chain $[1,15]$ of subgroups

$$
U(n) \supset U(n-1) \supset \ldots \supset U(2) \supset U(1) .
$$

The irreducible representations of the groups $U(m), 1 \leqslant m \leqslant n$, may be characterized by partitions $\left(\lambda_{1 m}, \lambda_{2 m} \ldots, \lambda_{m m}\right)$ where the $\lambda_{i m}$ are integers satisfying

$$
\lambda_{1 m} \geqslant \lambda_{2 m} \geqslant \ldots \geqslant \lambda_{m m} \geqslant 0 \text {. }
$$


The partitions of two groups $U(m+1)$ and $U(m)$ in the chain (39) are related by the inequalities

$$
\lambda_{1 m+1} \geqslant \lambda_{1 m} \geqslant \lambda_{2 m+1} \geqslant \lambda_{2 m} \geqslant \ldots \geqslant \lambda_{m m} \geqslant \lambda_{m+1 m+1}
$$

The set of partitions for the canonical chain (39) is most conveniently arranged into a Gelfand pattern [3] (see also Loebl [12]).

It is our aim in this section to obtain the reduced matrix elements of the $U(n)$ generators.

Maintaining the canonical subgroup decomposition in evidence at all stages we proceed recursively from $U(m)$ to $U(m+1)$ where only the operators $a_{m+1}^{i}$ and $a_{i}^{m+1}(i<m+1)$ need be considered, all other generators being known by hypothesis from the $U(m)$ calculation. The matrix of $a_{m+1}^{m+1}$ of course is diagonal with eigenvalue

$$
\sum_{i=1}^{m+1} \lambda_{i, m+1}-\sum_{i=1}^{m} \lambda_{i m} .
$$

As before, we let $\psi$ denote the $U(m)$ vector operator with components $\psi^{i}=a_{m+1}^{i}$. Then $\psi^{\dagger}$ is a contragredient vector operator with components $\psi_{i}^{\dagger}=a_{i}^{m+1}$.

From equations (36) and (37) we have

$$
\begin{aligned}
& \psi[r] \gamma_{r}^{-1} \psi^{\dagger}[r]=f_{r}, \\
& \psi^{\dagger}[r] \bar{\gamma}_{r}^{-1} \psi[r]=\bar{f}_{r},
\end{aligned}
$$

where the $U(m)$ invariants $\gamma_{r}$ and $\bar{\gamma}_{r}$ are expressible in terms of the $U(m+1)$ roots $\beta_{k}$ and the $U(m)$ roots $\alpha_{r}$ as in equations (30) and (35).

Rearranging these expressions we may write

where $\delta_{r}$ and $\delta_{r}$ are given by

$$
\begin{aligned}
& \psi[r] \psi^{\dagger}[r]=\delta_{r} f_{r} \\
& \psi^{\dagger}[r] \psi[r]=\delta_{r} f_{r}
\end{aligned}
$$

$$
\begin{aligned}
& \delta_{r}=(-1)^{n} \prod_{p=1}^{m+1}\left(\beta_{p}-\alpha_{r}\right) \prod_{l=1, l \neq r}^{m}\left(\alpha_{r}-\alpha_{l}-1\right)^{-1}, \\
& \delta_{r}=(-1)^{n} \prod_{p=1}^{m+1}\left(\beta_{p}-\alpha_{r}-1\right) \prod_{l=1, l \neq r}^{m}\left(\alpha_{r}-\alpha_{l}+1\right)^{-1} .
\end{aligned}
$$

By taking the trace of equations (40) and (41) we obtain

$$
\begin{aligned}
& \bar{\gamma}_{r}=\psi[r]^{i} \psi^{\dagger}[r]_{i}=\delta_{r} \operatorname{tr}\left[f_{r}\right], \\
& \gamma_{r}=\psi^{\dagger}[r]_{i} \psi[r]^{i}=\delta_{r} \operatorname{tr}\left[\bar{f}_{r}\right] .
\end{aligned}
$$


Note that by substituting for $\bar{\gamma}_{r}$ and $\delta_{r}$ we obtain

$$
\operatorname{tr}\left[f_{r}\right]=\bar{\gamma}_{r} \delta_{r}^{-1}=\prod_{l=1, l \neq r}^{m}\left(\frac{\alpha_{r}-\alpha_{l}-1}{\alpha_{r}-\alpha_{l}}\right)
$$

which is Green's trace formula.

We now claim that the $\delta_{r}(r=1, \ldots, n)$ are the squares of the reduced matrix elements of the $a_{m+1}^{i}$ while the $\delta_{r}$ are the squares of the reduced matrix elements of the $a_{i}^{m+1}$. To prove this result we follow the notation of Louck and Biedenharn [14].

Suppressing our $U(m+1)$ labels we let $\left|\begin{array}{c}\lambda \\ (m)\end{array}\right\rangle$ denote a Gelfand basis state contained in a representation of $U(m)$ with highest weight $\lambda$ where $(m)$ denotes a Gelfand pattern for the subgroup $U(m-1)$. We may then denote a maximal $U(m)$ state by the pattern $\left|\begin{array}{c}\lambda \\ (\lambda)\end{array}\right\rangle$ or, in shorthand notation, $|\lambda\rangle$. We shall find it convenient to introduce the weight $\Delta_{r}$ with 1 in the $r$ th position and zeros elsewhere,

$$
\Delta_{r}=[0, \ldots, 0,1,0, \ldots, 0] \quad(1 \text { in position } r) .
$$

We then have

$$
\begin{aligned}
\left\langle\begin{array}{c}
\lambda \\
(\lambda)
\end{array}\left|\psi^{\dagger}[r]_{i} \psi[r]^{i}\right| \begin{array}{c}
\lambda \\
(\lambda)
\end{array}\right\rangle= & \left|\left\langle\lambda+\Delta_{r}\|\psi\|\right\rangle\right|^{2} \\
& \times \sum_{i,(\mu)}\left\langle\begin{array}{c}
\lambda \\
(\lambda)
\end{array} ; \begin{array}{c|c}
10 & \lambda+\Delta_{r} \\
(\mu)
\end{array}\right\rangle\left\langle\left.\begin{array}{c}
\lambda+\Delta_{r} \\
(\mu)
\end{array}\right|_{i} ^{10} \begin{array}{c}
\lambda \\
(\lambda)
\end{array}\right\rangle,
\end{aligned}
$$

where the term outside the summation is the square of the reduced matrix element of the vector operator $\psi$ (the $a_{m+1}^{i}$ ). The terms

$$
\left\langle\begin{array}{cc|c}
\lambda & 10 & \lambda+\Delta_{r} \\
(\lambda) & i & (\mu)
\end{array}\right\rangle
$$

are Clebsch-Gordan coefficients corresponding to a tensor operator with IR label [10] (a vector operator) with components given by the index $i$.

Since $\psi^{\dagger}[r]_{i} \psi[r]^{i}$ is a $U(m)$ invariant, its eigenvalue is independent of the parameters of the $U(m-1)$ subgroup. So we may write

$$
\left\langle\begin{array}{c}
\lambda \\
(\lambda)
\end{array}\left|\psi^{\dagger}[r]_{i} \psi[r]^{i}\right| \begin{array}{c}
\lambda \\
(\lambda)
\end{array}\right\rangle=\frac{1}{D[\lambda]} \sum_{(\nu)}\left\langle\begin{array}{c}
\lambda \\
(\nu)
\end{array}\left|\psi^{\dagger}[r]_{i} \psi[r]^{i}\right| \begin{array}{c}
\lambda \\
(\nu)
\end{array}\right\rangle,
$$

where we have summed over all vectors in the representation space of $U(m)$ with highest weight $\lambda$ and then divided through by the dimension $D[\lambda]$ of the representation. 
Hence (44) may be written

$$
\begin{aligned}
& \left\langle\begin{array}{c}
\lambda \\
(\lambda)
\end{array}\left|\psi^{+}[r]_{i} \psi[r]^{i}\right| \begin{array}{c}
\lambda \\
(\lambda)
\end{array}\right\rangle=\left|\left\langle\lambda+\Delta_{r}\|\psi\| \lambda\right\rangle\right|^{2} \\
& \times \frac{1}{D[\lambda]} \sum_{i,(v),(\mu)} \\
& \times\left\langle\begin{array}{cc|c}
\lambda & 10 & \lambda+\Delta_{r} \\
(\nu) & i & (\mu)
\end{array}\right\rangle\left\langle\begin{array}{c|cc}
\lambda+\Delta_{r} & 10 & \lambda \\
(\mu) & ; & ; \\
i & (\nu)
\end{array}\right\rangle .
\end{aligned}
$$

From the orthogonality relations satisfied by Clebsch-Gordan coefficients (see Louck and Biedenharn [14] and Loebl [12]) we have

$$
\sum_{(\mu), i}\left\langle\begin{array}{c}
\lambda+\Delta_{r} \\
\left(\mu^{\prime}\right)
\end{array} \mid \begin{array}{cc}
10 & \lambda \\
i & (\mu)
\end{array}\right\rangle\left\langle\begin{array}{cc|c}
\lambda & 10 & \lambda+\Delta_{s} \\
(\mu) & i & i \\
\left(\nu^{\prime}\right)
\end{array}\right\rangle=\delta_{r s} \delta_{\left(\mu^{\prime}\right)\left(\nu^{\prime}\right)} .
$$

Substituting this relation into (45) gives the result

$$
\left\langle\begin{array}{c}
\lambda \\
(\lambda)
\end{array}\left|\psi^{\dagger}[r]_{i} \psi[r]^{i}\right| \begin{array}{c}
\lambda \\
(\lambda)
\end{array}\right\rangle=\frac{D\left[\lambda+\Delta_{r}\right]}{D[\lambda]}\left|\left\langle\lambda+\Delta_{r}\|\psi\| \lambda\right\rangle\right|^{2} .
$$

However, we also have (see Edwards [2])

$$
\operatorname{tr} \bar{f}_{r}=\frac{D\left[\lambda+\Delta_{r}\right]}{D[\lambda]} .
$$

Hence we may write

$$
\psi^{\dagger}[r]_{i} \psi[r]^{i}=\operatorname{tr} \bar{f}_{r}\left|\left\langle\lambda+\Delta_{r}\|\psi\| \lambda\right\rangle\right|^{2} .
$$

Comparing this expression with equation (43) we see that the invariants $\delta_{r}^{\ddagger}$ are our reduced matrix elements.

In a similar way we may show that the $\delta_{r}^{\ddagger}$ are the reduced matrix elements of the generators $a_{i}^{m+1}$. Since these invariants have been evaluated as a function of the $U(m+1)$ roots and $U(m)$ roots this enables us to evaluate all of the reduced matrix elements.

Finally, we note that the operators $\tilde{\psi}[r]$ and $\tilde{\psi}^{\dagger}[r]$ defined by

$$
\begin{gathered}
\tilde{\psi}[r]=\delta_{r}^{-\frac{1}{2}} \psi[r]=\psi[r] \gamma_{r}^{-\frac{1}{t}}, \\
\psi^{\dagger}[r]=\psi^{\dagger}[r] \delta_{r}^{-\frac{1}{2}}=\gamma_{r}^{-\frac{1}{4}} \psi^{\dagger}[r]
\end{gathered}
$$

are Wigner operators [14]. Hence from equation (36) we see that coupling together the Wigner operator $\tilde{\psi}[r]$ with its adjoint $\tilde{\psi}^{+}[r]$ gives us the $U(m)$ projectors $f_{r}$. 


\section{Extension to $O(n)$}

We now consider the extension of our previous results to the orthogonal subgroup of $U(n)$. By introducing a symmetric metric $g_{i j}$ we may take our $O(n)$ generators to be

$$
\alpha_{i j}=g_{i k} a_{j}^{k}-g_{j k} a_{i}^{k},
$$

where $a_{j}^{i}$ are the generators of $U(n)$. No loss of generality is in fact incurred by taking $g_{i j}=\delta_{i j}$. The advantage of this choice of metric is that we obtain a simple canonical subgroup embedding $O(n) \subset O(n+1)$.

So throughout the remainder of this paper we write our generators in the form

$$
\alpha_{j}^{i}=a_{j}^{i}-a_{i}^{j}=-\alpha_{i}^{j} .
$$

We then obtain the commutation relations

$$
\left[\alpha_{j}^{i}, \alpha_{l}^{k}\right]=\delta_{j}^{k} \alpha_{l}^{i}-\delta_{l}^{i} \alpha_{j}^{k}-\delta_{i}^{k} \alpha_{l}^{j}+\delta_{l}^{j} \alpha_{i}^{k} .
$$

As for $U(n)$ we introduce the $O(n)$ matrix $\alpha$ whose $(i, j)$ entry is the generator $\alpha_{j}^{i}$ Similarly we introduce its adjoint $\bar{\alpha}$ defined by

$$
\bar{\alpha}_{j}^{i}=-\alpha_{j}^{i} .
$$

We then define powers of the matrix $\alpha$ recursively by the formula

$$
\left(\alpha^{m}\right)_{j}^{i}=\alpha_{k}^{i}\left(\alpha^{m-1}\right)_{j}^{k}=\left(\alpha^{m-1}\right)_{k}^{i} \alpha_{j}^{k}
$$

which then enables us to define arbitrary polynomials in the matrix $\alpha$. In a similar way we may define polynomials in the matrix $\bar{\alpha}$.

It is easily shown, if $p(x)$ is any polynomial over the underlying field $F$, that we obtain the commutation relations

$$
\left[\alpha_{j}^{i}, p(\alpha)_{l}^{k}\right]=\delta_{j}^{k} p(\alpha)_{l}^{i}-\delta_{l}^{i} p(\alpha)_{j}^{k}-\delta_{i}^{k} p(\alpha)_{l}^{j}+\delta_{l}^{j} p(\alpha)_{i}^{k} .
$$

We take as a Cartan sub-algebra for the Lie algebra of $O(n)$ the vector space spanned by the operators

where

$$
h_{r}=-i \alpha_{2 r}^{2 r-1}, \quad r=1, \ldots, h,
$$

$$
h=\left[\begin{array}{l}
n \\
2
\end{array}\right]= \begin{cases}\frac{1}{2} n, & n \text { even } \\
\frac{1}{2}(n-1), & n \text { odd }\end{cases}
$$

It has been shown by Green and Bracken $[5,6]$ that the matrix $\alpha$ satisfies a polynomial identity of the form

$$
\prod_{r=1}^{n}\left(\alpha-\alpha_{r}\right)=0
$$


On a finite dimensional irreducible representation with highest weight $\left(\lambda_{1}, \ldots, \lambda_{h}\right)$ the operators $\alpha_{r}$ take constant values given by

$$
\begin{aligned}
\alpha_{r} & =\lambda_{r}+n-1-r, \\
\alpha_{n+1-r} & =r-1-\lambda_{r} \quad(r=1, \ldots, h),
\end{aligned}
$$

with

$$
\alpha_{h+1}=h \text { for } O(n=2 h+1) .
$$

The matrix $\bar{\alpha}$ satisfies the identity

$$
\prod_{r=1}^{n}\left(\bar{\alpha}-\bar{\alpha}_{r}\right)=0,
$$

where the $\bar{\alpha}_{r}$ are related to the $\alpha_{r}$ by

$$
\bar{\alpha}_{r}=\alpha_{n+1-r} .
$$

We now wish to proceed in analogy with $G L(n)$ but unfortunately the entries of the matrix $\alpha$ are not root space elements since our generators are not in Cartan form. We overcome this by applying a change of basis transformation to the matrix $\alpha$. This is effected by introducing an appropriate numerical matrix $M$ so that the matrix $M^{-1} \alpha M$ has entries consisting of $O(n)$ generators in their root space forms.

For $O(n=2 h)$ we take $M$ to be the unitary matrix with entries defined by

$$
\begin{aligned}
M_{i}^{2 i-1} & =\frac{1}{\sqrt{2}}=M_{n+1-i}^{2 i-1}, \\
M_{i}^{2 i} & =-\frac{i}{\sqrt{2}}=-M_{n+1-i}^{2 i} \quad(i=1, \ldots, h),
\end{aligned}
$$

all other entries being zero, that is

$$
M=\frac{1}{\sqrt{2}}\left(\begin{array}{rrrrrrrr}
1 & 0 & \ldots & \ldots & \ldots & \ldots & 0 & 1 \\
-i & 0 & \ldots & \ldots & \ldots & \ldots & 0 & i \\
0 & 1 & \ldots & \ldots & \ldots & \ldots & 1 & 0 \\
0 & -i & \ldots & \ldots & \ldots & \ldots & i & 0 \\
\vdots & \vdots & \ddots & & 0 & . . & \vdots & \vdots \\
0 & 0 & \ldots & 1 & l & \ldots & 0 & 0 \\
0 & 0 & \ldots & -i & i & \ldots & 0 & 0 \\
& & & \uparrow & \uparrow & & & \\
& & & h & h+1 & &
\end{array}\right)
$$


For $O(n=2 h+1)$ we add an extra row and column to give an additional nonzero entry $M_{h+1}^{n}=1$.

The inverse matrix $M^{-1}$ then has entries defined by

$$
\begin{aligned}
\left(M^{-1}\right)_{2 i-1}^{i} & =\frac{1}{\sqrt{2}}=\left(M^{-1}\right)_{2 i-1}^{n+1-i}, \\
\left(M^{-1}\right)_{2 i}^{i} & =\frac{i}{\sqrt{2}}=-\left(M^{-1}\right)_{2 i}^{n+1-i} \quad(i=1, \ldots, h),
\end{aligned}
$$

all other entries being zero except in the case when $n=2 h+1$ is odd where we have an additional non-zero entry

$$
\left(M^{-1}\right)_{n}^{h+1}=1 .
$$

Throughout the remainder of this paper we let $a$ denote the matrix

$$
a=M^{-1} \alpha M, \quad a_{f}^{i}=\left(M^{-1}\right)_{p}^{i} \alpha_{q}^{p} M_{j}^{q} .
$$

We define polynomials in the matrix $a$ as before. By repeated application of (49) we may show that

$$
p(a)=M^{-1} p(\alpha) M
$$

From this we see that the matrices $\alpha$ and $a$ satisfy the same characteristic identity (that is the characteristic identities remain unchanged by a change of basis).

We define the adjoint $\bar{a}$ of $a$ by

$$
\bar{a}_{i}^{j}=-a_{i}^{j}
$$

The adjoint $\bar{a}$ of $a$ is related to the adjoint $\bar{\alpha}$ of $\alpha$ by

where

$$
\bar{a}_{j}^{i}=\bar{M}_{j}^{p} \bar{\alpha}_{p}^{q}(\bar{M})_{q}^{-1 i}
$$

$$
\bar{M}_{p}^{q}=M_{p}^{q}, \quad(\bar{M})_{p}^{-1 q}=M_{p}^{-1 q} .
$$

More generally if $p(x)$ is any polynomial over the field $F$ we have

$$
p(\bar{a})=\bar{M} p(\bar{\alpha}) \bar{M}^{-1} .
$$

Hence the matrices $\bar{a}$ and $\bar{\alpha}$ also satisfy the same identity.

We now evaluate the commutators $\left[a_{j}^{i}, a_{l}^{k}\right]$. We have

$$
\left[a_{j}^{i}, a_{l}^{k}\right]=\left(M^{-1}\right)_{p}^{i} M_{j}^{q}\left(M^{-1}\right)_{r}^{k} M_{l}^{\ell}\left[\alpha_{q}^{p}, \alpha_{l}^{r}\right] .
$$

Using the commutation relations (47) the right-hand side becomes

$$
\delta_{j}^{p} a_{q}^{i}-\delta_{q}^{i} a_{j}^{p}-\left(M^{-1}\right)_{r}^{i}\left(M^{-1}\right)_{r}^{p} M_{j}^{m} M_{q}^{k} \alpha_{k}^{m}+M_{j}^{k} M_{q}^{k}\left(M^{-1}\right)_{l}^{i}\left(M^{-1}\right)_{r}^{p} \alpha_{l}^{r} .
$$


Now $M_{j}^{k} M_{q}^{k}$ is the inner product between the $j$ th and $q$ th columns of the matrix $M$. Hence we have

$$
M_{j}^{k} M_{q}^{k}=\delta_{j, n+1-q}
$$

Similarly

$$
\left(M^{-1}\right)_{r}^{i}\left(M^{-1}\right)_{r}^{p}=\delta_{i, n+1-p}
$$

Also, from (49), we have

$$
\begin{gathered}
\alpha_{k}^{m} M_{q}^{k}=M_{k}^{m} a_{q}^{k}, \\
\left(M^{-1}\right)_{r}^{p} \alpha_{l}^{r}=a_{r}^{p}\left(M^{-1}\right)_{l}^{r} .
\end{gathered}
$$

Thus we obtain the commutation relations

$$
\left[a_{j}^{i}, a_{q}^{p}\right]=\delta_{j}^{p} a_{q}^{i}-\delta_{q}^{i} a_{j}^{p}-\delta_{n+1-i}^{p} a_{q}^{n+1-j}+\delta_{q}^{n+1-j} a_{n+1-i}^{p} .
$$

More generally, using (48) and (50), we obtain

$$
\left[a_{j}^{i}, p(a)_{q}^{p}\right]=\delta_{j}^{p} p(a)_{q}^{i}-\delta_{q}^{i} p(a)_{j}^{p}-\delta_{n+1-i}^{p} p(a)_{p}^{n+1-j}+\delta_{q}^{n+1-j} p(a)_{n+1-i}^{p}
$$

We now note that the diagonal entries of the matrix $a$ are given by

$$
\begin{aligned}
a_{r}^{r} & =h_{r}, \\
a_{n+1-r}^{n+1-r} & =-h_{r} \quad(r=1, \ldots, h),
\end{aligned}
$$

with

$$
a_{h+1}^{h+1}=0 \text { for } O(n=2 h+1)
$$

From this and the commutation relations (51) we see that, for $i<j \leqslant h, a_{n+1-i}^{n+1-j}$ and $a_{j}^{i}$ are both positive roots of weight $e_{i}-e_{j}$. Similarly the $a_{n+1-j}^{n+1-i}$ and $a_{i}^{j}$ are negative roots of weight $e_{j}-e_{i}$. For $i, j=1, \ldots, h$ the entries $a_{n+1-j}^{i}$ are positive roots of weight $e_{i}+e_{j}$ while the $a_{i}^{n+1-j}$ are negative roots of weight $-\left(e_{i}+e_{j}\right)$.

Finally, for $O(n=2 h+1)$ we have the additional positive roots $a_{h+1}^{i}$ and $a_{n+1-i}^{h+1}(i=1, \ldots, h)$ of weight $e_{i}$ and negative roots $a_{i}^{h+1}$ and $a_{h+1}^{n+1-i}$ of weight $-e_{i}$.

It is easily checked that these roots agree with those obtained by Wong [18] (except that his C.S.A. is minus ours). The advantage of writing the root space elements in this form is that we maintain the simplicity of our commutation relations and furthermore the analogy with $U(n)$ is clearer.

Note also that the positive roots appear above the diagonal of the matrix $a$ while the negative roots appear below the diagonal. Our analogy with $U(n)$ is now complete and we may proceed as before. 
Now let $v_{0}$ be an $O(n)$ maximal weight vector of arbitrary weight $\lambda$. Introducing a set of polynomials

$$
\begin{aligned}
& p_{r}(x)=\prod_{k=r}^{n}\left(x-\lambda_{k}-n+1+k\right), \\
& q_{r}(x)=\prod_{k=1}^{r}\left(x+\lambda_{k}+1-k\right),
\end{aligned}
$$

where we define $\lambda_{r}$ for $r>h$ by

$$
\lambda_{n+1-r}=1-\lambda_{r}, \quad r=1, \ldots, h,
$$

we may verify propositions (1) and (2) as before. From this it is then easily shown that Green's identities for $O(n)$ hold on arbitrary representations generated by a maximal weight vector.

As for $U(n)$ we may construct a set of projectors

$$
\begin{aligned}
& f_{r}=\prod_{l \neq r}\left(\frac{a-\alpha_{l}}{\alpha_{r}-\alpha_{l}}\right), \\
& f_{r}=\prod_{l \neq r}\left(\frac{\bar{a}-\bar{\alpha}_{l}}{\bar{\alpha}_{r}-\bar{\alpha}_{l}}\right),
\end{aligned}
$$

which satisfy

$$
\tilde{f}_{r} \bar{f}_{k}=\delta_{r k} \bar{f}_{k}, \quad f_{r} f_{k}=\delta_{r k} f_{k}
$$

and

$$
\sum_{r=1}^{n}\left(f_{r}\right)_{j}^{i}=\delta_{j}^{i}, \quad \sum_{r=1}^{n}\left(\bar{f}_{r}\right)_{j}^{i}=\delta_{j}^{i}
$$

Then, if $p(x)$ is any polynomial over the field $F$, we may write

$$
\begin{aligned}
& p(a)=\sum_{r=1}^{n} p\left(\alpha_{r}\right) f_{r}, \\
& p(\bar{a})=\sum_{r=1}^{n} p\left(\bar{\alpha}_{r}\right) \tilde{f}_{r} .
\end{aligned}
$$

\section{Casimir invariants for $O(n)$}

As for $U(n)$ we obtain a full set of invariants for $O(n)$ by taking traces in the polynomials of the matrix $a$. We proceed in analogy with $U(n)$ to verify Green's trace formula for the orthogonal groups for arbitrary weights.

Let $v_{0}$ be a maximal $O(n)$ weight vector of weight $\lambda=\left(\lambda_{1}, \ldots, \lambda_{h}\right)$. We consider now the operators $p_{i}(k)$ and $p_{i, j}(k)$ defined as in equation (11) with the $O(n)$ roots 
$\alpha_{r}$ taking values given by

with

$$
\begin{aligned}
\alpha_{r} & =\lambda_{r}+n-1-r, \\
\alpha_{n+1-r} & =r-1-\lambda_{r} \quad(r=1, \ldots, h),
\end{aligned}
$$

$$
\alpha_{h+1}=h \text { for } O(n=2 h+1) \text {. }
$$

As before, we let $\rho_{i}(k)$ and $\rho_{i, j}(k)$ be the respective eigenvalues of these operators when acting on the maximal weight vector $v_{0}$. It is easily verified that the $\rho_{i}(k)$ satisfy the difference equation

$$
\rho_{i}(r)=\left(\alpha_{r}-\alpha_{i}\right) \rho_{i+1}(r)-\sum_{k>r} \rho_{i+1}(k)+ \begin{cases}0, & r>h, \\ \rho_{i+1}(n+1-r), & r<h .\end{cases}
$$

The $\rho_{i, j}(r)$ for $i<j-1$ satisfy

$$
\rho_{i, j}(r)=\left(\alpha_{r}-\alpha_{i}\right) \rho_{i+1, j}(r)-\sum_{k>r} \rho_{i+1, j}(k)+ \begin{cases}0, & r>h, \\ \rho_{i+1, j}(n+1-r), & r<h\end{cases}
$$

and when $i=j-1$ we obtain

$$
\rho_{j-1, j}(r)=\left(\alpha_{r}-\alpha_{j-1}\right) \rho_{j+1}(r)-\sum_{k>r} \rho_{j+1}(k)+ \begin{cases}0, & r>h, \\ \rho_{j+1}(n+1-r), & r<h .\end{cases}
$$

Proceeding as before we obtain the following formulae:

$$
O(n=2 h)
$$

$$
\begin{aligned}
& \left(f_{r}\right)_{k}^{k} v_{0}= \begin{cases}\prod_{l>k}\left(\frac{\alpha_{r}-\alpha_{l}-1+\delta_{l, n+1-r}}{\alpha_{r}-\alpha_{l}+\delta_{r l}}\right) v_{0}, & k \leqslant r, \\
0, \quad k>r,\end{cases} \\
& \left(\bar{f}_{r}\right)_{k}^{k} v_{0}= \begin{cases}\prod_{>k}\left(\frac{\bar{\alpha}_{r}-\bar{\alpha}_{l}-1+\delta_{l, n+1-r}}{\bar{\alpha}_{r}-\bar{\alpha}_{l}+\delta_{r l}}\right) v_{0}, & k \geqslant r, \\
0, & k<r .\end{cases}
\end{aligned}
$$

$O(n=2 h+1)$

$$
\begin{aligned}
& \left(f_{r}\right)_{k}^{k} v_{0}= \begin{cases}\prod_{l>k}\left(\frac{\alpha_{r}-\alpha_{l}-1+\delta_{l, h+1}-\delta_{l, n+1-r}}{\alpha_{r}-\alpha_{l}+\delta_{r l}}\right) v_{0}, & k \leqslant r, \\
0, & k>r .\end{cases} \\
& \left(f_{r}\right)_{k}^{k} v_{0}= \begin{cases}\prod_{l<k}\left(\frac{\bar{\alpha}_{r}-\bar{\alpha}_{l}-1+\delta_{l, h+1}-\delta_{l, n+1-r}}{\bar{\alpha}_{r}-\bar{\alpha}_{l}+\delta_{r l}}\right) v_{0}, & k \geqslant r, \\
0, & k<r .\end{cases}
\end{aligned}
$$


Summing these equations over $k$ from 1 to $n$ we obtain the formulae

$$
\begin{aligned}
& \operatorname{tr} f_{r}=\prod_{l \rightarrow r}\left(\frac{\alpha_{r}-\alpha_{l}-1+\delta_{l, n+1-r}}{\alpha_{r}-\alpha_{l}}\right), \quad n=2 h, \\
& \operatorname{tr} f_{r}=\prod_{l \rightarrow r}\left(\frac{\alpha_{r}-\alpha_{l}-1+\delta_{l, h+1}-\delta_{l, n+1-r}}{\alpha_{r}-\alpha_{l}}\right), \quad n=2 h+1, \quad r \neq h+1 .
\end{aligned}
$$

When $n=2 h+1$ and $r=h+1$ we obtain

$$
\operatorname{tr} f_{h+1}=\prod_{l \neq h+1}\left(\frac{\alpha_{h+1}-\alpha_{l}-1}{\alpha_{h+1}-\alpha_{l}}\right)=1,
$$

where we have used $\alpha_{h+1}-\alpha_{1}-1=\alpha_{n+1-1}-\alpha_{h+1}$ for $n$ odd and $l \neq h+1$. This shows that Green's trace formulae for $O(n)$ extend to arbitrary weights.

\section{Reduced matrix elements for $O(n)$}

With respect to our $O(n)$ generators $a_{j}^{i}$ we define an $O(n)$ vector operator as an operator with $n$ components $\psi^{i}$ which satisfy

$$
\left[a_{j}^{i}, \psi^{k}\right]=\delta_{j}^{k} \psi^{i}-\delta_{n+1-i}^{k} \psi^{n+1-i} .
$$

Similarly we define an $O(n)$ contragredient vector operator with components $\phi_{i}$ which transform according to

$$
\left[a_{j}^{i}, \phi_{k}\right]=-\delta_{k}^{i} \phi_{j}+\delta_{n}^{k+1-i} \phi_{n+1-i} .
$$

With respect to our old generators $\alpha_{j}^{i}$ this is equivalent to choosing components $\tilde{\psi}_{j}^{i}$ and $\tilde{\phi}_{i}$ which satisfy

$$
\begin{aligned}
& {\left[\alpha_{j}^{i}, \tilde{\psi}_{j}^{k}\right]=\delta_{j}^{k} \psi^{i}-\delta_{i}^{k} \tilde{\psi}^{i},} \\
& {\left[\alpha_{j}^{i}, \tilde{\phi}_{k}\right]=-\delta_{k}^{i} \tilde{\phi}_{j}+\delta_{k}^{j} \tilde{\phi}_{i} .}
\end{aligned}
$$

If $\tilde{\psi}$ is a vector operator with components $\tilde{\psi}^{i}$ satisfying (53') we may transform $\tilde{\psi}$ into a vector operator $\psi$ with components $\psi^{i}$ satisfying (53) by applying the matrix $M^{-1}$,

$$
\psi^{i}=\left(M^{-1}\right)_{j}^{i} \tilde{\psi}^{j}
$$

Their components are therefore related by

with

$$
\begin{aligned}
\psi^{i} & =\tilde{\psi}^{2 i-1}+i \tilde{\psi}^{2 i}, \\
\psi^{n+1-i} & =\tilde{\psi}^{2 i-1}-i \tilde{\psi}^{2 i} \quad(i=1, \ldots, h),
\end{aligned}
$$

$$
\psi^{h+1}=\tilde{\psi}^{n} \quad \text { for } n=2 h+1
$$


Similarly if $\tilde{\phi}$ is a contragredient vector operator transforming according to (54') then

$$
\phi_{i}=\Phi_{j} M_{i}^{i}
$$

transforms according to (54). This shows the relation between vector operators in both choices of basis for the Lie algebra of $O(n)$.

Following the same notation as before, an $O(n)$ vector operator $\psi$ may be resolved into a sum of vector operators $\psi[r]$,

$$
\psi=\sum_{r=1}^{n} \psi[r]
$$

where each $\psi[r]$ satisfies

$$
\begin{aligned}
\lambda_{k} \psi[r] & =\psi[r]\left(\lambda_{k}+\delta_{k r}\right), \\
\lambda_{k} \psi[n+1-r] & =\psi[n+1-r]\left(\lambda_{k}-\delta_{k r}\right) \quad(r=1, \ldots, h),
\end{aligned}
$$

with

$$
\lambda_{k} \psi[h+1]=\psi[h+1] \lambda_{k} \text { for } n=2 h+1 \text {, }
$$

where $\lambda=\left(\lambda_{1}, \lambda_{2}, \ldots, \lambda_{h}\right)$ is the labelling operator for $O(n)$.

These shift components may be constructed by application of the projectors $f_{r}$ and $f_{r}$,

$$
\psi[r]=f_{r} \psi=\psi \bar{f}_{r} .
$$

Similarly if $\phi$ is a contragredient vector operator we may resolve $\phi$ into components $\phi[r]$ which satisfy

$$
\begin{aligned}
\lambda_{k} \phi[r] & =\phi[r]\left(\lambda_{k}-\delta_{k r}\right), \\
\lambda_{k} \phi[n+1-r] & =\phi[n+1-r]\left(\lambda_{k}+\delta_{k r}\right) \quad(r=1, \ldots, h),
\end{aligned}
$$

with

$$
\lambda_{k} \phi[h+1]=\phi[h+1] \lambda_{k} \text { for } n=2 h+1 \text {. }
$$

The shift components $\phi[r]$ of $\phi$ may also be constructed by application of the $f_{r}$ and $\tilde{f}_{r}$,

$$
\phi[r]=\tilde{f}_{r} \phi=\phi f_{r} .
$$

Now let $\alpha_{j}^{i}$ be the $O(n+1)$ generators defined by (46). We denote the $O(n+1)$ matrix whose $(i, j)$ entry is the generator $\alpha_{j}^{i}$ by $\beta$. As for $U(n) \subset U(n+1)$, the $O(n)$ matrix $\alpha$ is canonically embedded in the $O(n+1)$ matrix $\beta$. The first $n$ entries of the last column of the matrix $\beta$ (that is the $\alpha_{n+1}^{i}$ ) constitute an $O(n)$ vector operator while the first $n$ entries of the last row (that is the $\alpha_{i}^{n+1}$ ) gives us an $O(n)$ contragredient vector operator. However, the generators are not in Cartan form. If we put the generators of $O(n+1)$ into Cartan form we lose the canonical embedding of the $O(n)$ matrix inside the $O(n+1)$ matrix when $n$ is odd. 
We overcome this by putting the generators of $O(n+1)$ into their whight space forms with respect to the Cartan subalgebra of $O(n)$. This leads us to consider the matrix $\tilde{M}$ defined by

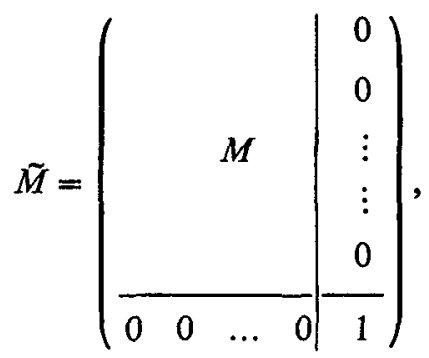

where $M$ is the transformation matrix introduced earlier.

We now consider the $O(n+1)$ matrix defined by

$$
b=\hat{M}^{-1} \beta \hat{M} .
$$

Then the $O(n)$ matrix $a=M^{-1} \alpha M$ is canonically embedded in the $O(n+1)$ matrix $b$,

$$
b=\left(\begin{array}{ccc|c} 
& & & b_{n+1}^{1} \\
& & & \vdots \\
& a & & \vdots \\
& & & b_{n+1}^{n} \\
\hline b_{1}^{n+1} & \ldots & b_{n}^{n+1} & 0
\end{array}\right)
$$

The entries $b_{i}^{n+1}, b_{n+1}^{i}$ are given by

$$
\begin{aligned}
& b_{n+1}^{i}=\left(M^{-1}\right)_{q}^{i} \alpha_{n+1}^{q}, \\
& b_{i}^{n+1}=\alpha_{q}^{n+1} \dot{M}_{i}^{q} .
\end{aligned}
$$

Hence the $b_{n+1}^{i}$ constitute an $O(n)$ vector operator transforming according to (53). Similarly the $b_{i}^{n+1}$ constitute an $O(n)$ contragredient vector operator transforming according to (54).

Let us denote the $O(n)$ vector operator with components $b_{n+1}^{i}$ by $\psi$. Now we note that the $O(n)$ generators $\alpha_{j}^{i}$ satisfy the Hermiticity property

$$
\left(\alpha_{j}^{i}\right)^{\dagger}=\alpha_{i}^{j}
$$

Since the matrices $M$ and $\hat{M}$ are unitary it is easily checked that the matrfees $a$ and $b$ also satisfy

$$
\left(a_{j}^{i}\right)^{\dagger}=a_{i}^{j}, \quad\left(b_{j}^{i}\right)^{\dagger}=b^{i}
$$


More generally if $p(x)$ is any polynomial over the field $F$ then we have

$$
\left(p(a)_{l}^{k}\right)^{\dagger}=p(a)_{k}^{l}, \quad\left(p(b)_{l}^{k}\right)^{\dagger}=p(b)_{k}^{l} .
$$

From this we have, in particular, $\left(b_{n+1}^{i}\right)^{\dagger}=b_{i}^{n+1}$. So we let $\psi^{\dagger}$ denote the contragredient vector operator with components

$$
\psi_{i}^{\dagger}=b_{i}^{n+1}
$$

We write our $O(n+1)$ identity in the form

$$
\prod_{r=1}^{n+1}\left(b-\beta_{r}\right)=0
$$

and denote the $O(n+1)$ projectors by

$$
\begin{aligned}
& g_{r}=\prod_{p=1, p \neq r}^{n+1}\left(\frac{b-\beta_{p}}{\beta_{r}-\beta_{p}}\right), \\
& \bar{g}_{r}=\prod_{p=1, p \neq r}^{n+1}\left(\frac{\bar{b}-\bar{\beta}_{r}}{\bar{\beta}_{r}-\bar{\beta}_{p}}\right),
\end{aligned}
$$

where $\beta_{r}=\beta_{n+2-r}$.

Proceeding as for $U(n)$ we may apply the $O(n+1)$ characteristic identity to give

$$
\begin{aligned}
& \left(g_{p}\right)_{n+1}^{i}=\sum_{r=1}^{n}\left(\beta_{p}-\alpha_{r}\right)^{-1} \psi[r]^{i} \varepsilon_{p}, \\
& \left(g_{p}\right)_{i}^{n+1}=\sum_{r=1}^{n} \varepsilon_{p} \psi^{\dagger}[r]_{i}\left(\beta_{p}-\alpha_{r}\right)^{-1},
\end{aligned}
$$

where

$$
\varepsilon_{p}=\left(g_{p}\right)_{n+1}^{n+1}
$$

Rearranging these expressions we obtain

$$
\begin{aligned}
& \left(g_{p}\right)_{n+1}^{i}=\sum_{r=1}^{n} \psi[r]^{i}\left(\beta_{p}-\alpha_{r}-\eta_{r}\right)^{-1} \varepsilon_{p} \\
& \left(g_{p}\right)_{i}^{n+1}=\sum_{r=1}^{n} \varepsilon_{p}\left(\beta_{p}-\alpha_{r}-\eta_{r}\right)^{-1} \psi^{+}[r]_{i}
\end{aligned}
$$

where

$$
\eta_{r}=\eta_{n+1-r}=1 \text { for } r=1, \ldots, h
$$

with

$$
\eta_{h+1}=0 \text { for } n=2 h+1
$$


From these expressions we obtain the results

$$
\begin{gathered}
\varepsilon_{p}=\prod_{q=1, q \neq p}^{n+1}\left(\beta_{p}-\beta_{q}\right)^{-1} \prod_{r=1}^{n}\left(\beta_{p}-\alpha_{r}-\eta_{r}\right), \\
\psi^{+}[r]_{i} \psi[r]^{i}=\gamma_{r}=(-1)^{n} \prod_{p=1}^{n+1}\left(\beta_{p}-\alpha_{r}-\eta_{r}\right) \prod_{l=1,1+r}^{n}\left(\alpha_{r}-\alpha_{l}+\eta_{r}-\eta_{l}\right)^{-1} .
\end{gathered}
$$

Similarly, by applying the $O(n+1)$ adjoint identity, we obtain

$$
\begin{aligned}
& \bar{\varepsilon}_{p}=\left(\bar{g}_{p}\right)_{n+1}^{n+1}=\prod_{q=1, q \neq p}^{n+1}\left(\bar{\beta}_{p}-\bar{\beta}_{q}\right)^{-1} \prod_{l=1}^{n}\left(\bar{\beta}_{p}-\bar{\alpha}_{r}-\eta_{r}\right), \\
& \psi[r]^{i} \psi^{\dagger}[r]_{i}=\bar{\gamma}_{r}=(-1)^{n} \prod_{p=1}^{n+1}\left(\bar{\beta}_{p}-\bar{\alpha}_{r}-\eta_{r}\right) \prod_{l=1, l \neq r}^{n}\left(\bar{\alpha}_{r}-\bar{\alpha}_{l}+\eta_{r}-\eta_{l}\right)^{-1} .
\end{aligned}
$$

Finally, we have

$$
\begin{aligned}
& \psi^{\dagger}[r] \bar{\gamma}_{r}^{-1} \psi[r]=f_{r}, \\
& \psi[r] \gamma_{r}^{-1} \psi^{\dagger}[r]=f_{r},
\end{aligned}
$$

which gives the spectral resolutions

$$
\begin{aligned}
& p(a)=\sum_{r=1}^{n} p\left(\alpha_{r}\right) \psi[r] \gamma_{r}^{-1} \psi^{+}[r], \\
& p(\bar{a})=\sum_{r=1}^{n} p\left(\bar{\alpha}_{r}\right) \psi^{\dagger}[r] \bar{\gamma}_{r}^{-1} \psi[r] .
\end{aligned}
$$

Rearranging equations (55) allows us to write

where

$$
\begin{aligned}
& \psi^{\dagger}[r] \psi[r]=\delta_{r} \tilde{f}_{r}, \\
& \psi[r] \psi^{\dagger}[r]=\delta_{r} f_{r},
\end{aligned}
$$

$$
\begin{aligned}
& \delta_{r}=(-1)^{n} \prod_{p=1}^{n+1}\left(\bar{\beta}_{p}-\bar{\alpha}_{r}\right) \prod_{l=1, l \neq r}^{n}\left(\bar{\alpha}_{r}-\bar{\alpha}_{l}-\eta_{l}-\delta_{l, n+1-r}\right)^{-1}, \\
& \delta_{r}=(-1)^{n} \prod_{p=1}^{n+1}\left(\beta_{p}-\alpha_{r}\right) \prod_{l=1, l \neq r}^{n}\left(\alpha_{r}-\alpha_{l}-\eta_{l}-\delta_{l, n+1-r}\right)^{-1} .
\end{aligned}
$$

Taking the trace of the above formulae we obtain

$$
\begin{aligned}
& \operatorname{tr} f_{r}=\bar{\gamma}_{r} \delta_{r}^{-1}, \\
& \operatorname{tr} \bar{f}_{r}=\gamma_{r} \delta_{r}^{-1} .
\end{aligned}
$$

As for $U(n)$ the $\delta_{r}$ and $\delta_{r}$ are the squares of the reduced matrix elements of $\psi$ and $\psi^{\dagger}$ respectively. Note that the reduced matrix elements of $\psi$ remain unchanged by a basis transformation. Hence $\delta_{r}$ and $\delta_{r}$ are the reduced matrix elements of the $\alpha_{m+1}^{i}$ and $\alpha_{i}^{m+1}$ respectively. 
Proceeding recursively via the canonical chain

$$
O(n) \supset O(n-1) \supset \ldots \supset O(2),
$$

we see that we have in fact obtained the reduced matrix elements of all our $O(n)$ generators.

For future reference we write down some of our previous formulae for the cases $n$ odd and $n$ even separately.

$O(n=2 h)$

In this case we have $\eta_{r}=1$ and $\bar{\alpha}_{r}-\bar{\alpha}_{l}=\alpha_{l}-\alpha_{r}$.

$$
\begin{aligned}
& \gamma_{r}=\bar{\gamma}_{n+1-r}=(-1)^{n} \prod_{p=1}^{n+1}\left(\beta_{p}-\alpha_{r}-1\right) \prod_{l \neq r}\left(\alpha_{r}-\alpha_{l}\right)^{-1}, \\
& \delta_{r}=\delta_{n+1-r}=(-1)^{n} \prod_{p=1}^{n+1}\left(\beta_{p}-\alpha_{r}\right) \prod_{l \neq r}\left(\alpha_{r}-\alpha_{l}-1-\delta_{l, n+1-r}\right)^{-1} .
\end{aligned}
$$

Note that substituting for $\bar{\gamma}_{r}$ and $\delta_{r}$ from these expressions we obtain

$$
\operatorname{tr} f_{r}=\prod_{p=1}^{n+1}\left(\frac{\beta_{p}-\alpha_{r}-\delta_{p, h+1}}{\beta_{p}-\alpha_{r}}\right) \prod_{l \neq r}\left(\frac{\alpha_{r}-\alpha_{l}-1-\delta_{l, n+1-r}}{\alpha_{r}-\alpha_{l}}\right),
$$

where we have used

$$
\bar{\alpha}_{r}-\bar{\beta}_{p}=\beta_{p}-\alpha_{r}-1-\delta_{p, h+1} \quad \text { for } n=2 h .
$$

But we know

$$
\prod_{p=1}^{n+1}\left(\frac{\beta_{p}-\alpha_{r}-\delta_{p, h+1}}{\beta_{p}-\alpha_{r}}\right)=\frac{h-\alpha_{r}-1}{h-\alpha_{r}}=\prod_{l \neq r}\left(\frac{\alpha_{r}-\alpha_{l}-1+\delta_{l, n+1-r}}{\alpha_{r}-\alpha_{l}-1-\delta_{l, n+1-r}}\right)
$$

and hence we get

$$
\operatorname{tr} f_{r}=\prod_{l \neq r}\left(\frac{\alpha_{r}-\alpha_{l}-1+\delta_{l, n+1-r}}{\alpha_{r}-\alpha_{l}}\right),
$$

which is Green's trace formula.

$$
O(n=2 h+1)
$$

In this case we have $\eta_{r}=1-\delta_{r, h+1}$ and

$$
\begin{aligned}
\bar{\beta}_{p}-\bar{\alpha}_{r}-\eta_{r} & =\alpha_{r}-\beta_{p}, \quad r \neq h+1, \\
\bar{\alpha}_{r}-\bar{\alpha}_{l}+\eta_{l}-\eta_{r} & =\alpha_{l}-\alpha_{r}, \quad r \neq h+1,
\end{aligned}
$$


from which we obtain, for $r \neq h+1$,

$$
\begin{aligned}
& \bar{\gamma}_{r}=\gamma_{n+1 \rightarrow r}=(-1)^{n} \prod_{p=1}^{n+1}\left(\beta_{p}-\alpha_{r}\right) \prod_{l+r}\left(\alpha_{r}-\alpha_{l}\right)^{-1}, \\
& \delta_{r}=\delta_{n+1 \rightarrow r}=(-1)^{n} \prod_{p=1}^{n+1}\left(\beta_{p}-\alpha_{r}\right) \prod_{l+r}\left(\alpha_{r}-\alpha_{l}-1+\delta_{l, h+1}-\delta_{l, n+1 \rightarrow r}\right)^{-1} .
\end{aligned}
$$

In this case we obtain

$$
\operatorname{tr} f_{r}=\bar{\gamma}_{r} \delta_{r}^{-1}=\prod_{l \neq r}\left(\frac{\alpha_{r}-\alpha_{l}-1+\delta_{l, h+1}-\delta_{l, n+1-r}}{\alpha_{r}-\alpha_{l}}\right)
$$

as required.

Finally, when $r=h+1$, we have

$$
\gamma_{h+1}=\bar{\gamma}_{h+1}=\delta_{h+1}=\delta_{h+1}=\prod_{p=1}^{n+1}\left(\beta_{p}-h\right) \prod_{l \neq h+1}\left(h-\alpha_{l}-1\right)^{-1},
$$

which gives

$$
\operatorname{tr} f_{h+1}=\operatorname{tr} f_{h+1}=1
$$

\section{Appendix}

Let $g_{k}$ denote the $g l(n+1, F)$ projector

$$
g_{k}=\prod_{p=1, p \neq k}^{n+1}\left(\frac{b-\beta_{p}}{\beta_{k}-\beta_{p}}\right)
$$

We prove here that

$$
\left(g_{k}\right)_{j}^{i}=\left(g_{k}\right)_{n+1}^{i}\left(\varepsilon_{k}\right)^{-1}\left(g_{k}\right)_{j}^{n+1} .
$$

A proof of this result based on more general considerations can be found in Green [7]. However, for the sake of completeness, we present a proof here which relies explicitly on the $g l(n, F)$ commutation relations. This method also extends to $O(n)$.

Throughout we shall let $\left(\beta_{p}-a\right)^{-1}$ denote the matrix

$$
\left(\beta_{p}-a\right)^{-1}=\sum_{r=1}^{n}\left(\beta_{p}-\alpha_{r}\right)^{-1} f_{r}
$$

where $f_{r}$ is the $g l(n, F)$ projector as defined by equation (7).

From equation (22) we have

where

$$
\left(g_{k}\right)_{n+1}^{i}=\left(\beta_{k}-a\right)_{j}^{-1 i} a_{n+1}^{j} \varepsilon_{k}
$$

Now

$$
\varepsilon_{k}=g_{k_{n+1}^{n+1}}^{n+1}
$$

or

$$
\left[a_{j}^{n+1},\left(g_{k}\right)_{n+1}^{i}\right]=\delta_{j}^{i} \varepsilon_{k}-\left(g_{k}\right)_{j}^{i}
$$

$$
\left(g_{k}\right)_{j}^{i}=\delta_{j}^{i} \varepsilon_{k}-\left[a_{j}^{n+1},\left(g_{k}\right)_{n+1}^{i}\right] \text {. }
$$


On the other hand, evaluating the commutator $\left[a_{j}^{n+1},\left(g_{k}\right)_{n+1}^{i}\right]$ using (56) we obtain

$$
\left[a_{j}^{n+1},\left(g_{k}\right)_{n+1}^{i}\right]=\delta_{j}^{i} \varepsilon_{k}-\left(\beta_{k}-a\right)_{l}^{-1 i} a_{n+1}^{l}\left(g_{k}\right)_{j}^{n+1},
$$

where we have used

$$
\left[a_{j}^{n+1},\left(\beta_{k}-a\right)_{l}^{-1}\right]=-\left(\beta_{k}-a\right)_{p}^{-1}\left[a_{j}^{n+1},\left(\beta_{k}-a\right)_{q}^{p}\right]\left(\beta_{k}-a\right)_{l}^{-1 q} .
$$

Substituting this into (57) gives

$$
\left(g_{k}\right)_{j}^{i}=\left(\beta_{k}-a\right)_{l}^{-1 i} a_{n+1}^{l}\left(g_{k}\right)_{j}^{n+1}
$$

and the result follows from (56).

In view of equation (21) it is immediate that

$$
\sum_{k=1}^{n+1}\left(g_{k}\right)_{n+1}^{i} \varepsilon_{k}^{-1}\left(g_{k}\right)_{j}^{n+1}=\delta_{j}^{i} .
$$

\section{Acknowledgements}

The author would like to thank Professors H. S. Green and S. A. Edwards for several enlightening discussions which were invaluable in the section on reduced matrix elements. He also acknowledges the financial support of a Commonwealth Postgraduate Research Award.

\section{References}

[1] G. E. Baird and L. C. Biedenharn, J. Math. Phys. 4 (1963), 1449.

[2] S. A. Edwards, "A new approach to the eigenvalues of the Gel'fand invariants for the unitary, orthogonal and symplectic groups", Univ. Adelaide preprint.

[3] I. M. Gel'fand and M. L. Zetlin, Dokl. Akad. Nauk SSSR 71 (1950), 825.

[4] M. D. Gould, J. Austral. Math. Soc. B 20 (1978), (to appear).

[5] H. S. Green, J. Math. Phys. 12 (1971), 2106.

[6] A. J. Bracken and H. S. Green, J. Math. Phys. 12 (1971), 2099.

[7] H. S. Green, J. Austral. Math. Soc. B 19 (1975), 129.

[8] H. S. Green, C. A. Hurst and Y. Ilamed, J. Math. Phys. 17 (1976), 1376.

[9] K. C. Hannabuss, "Characteristic equations for semisimple Lie groups", Math. Inst. Oxford preprint (1972).

[10] J. E. Humphreys, Introduction to Lie algebras and representation theory (New YorkHeidelberg-Berlin: Springer-Verlag, 1972).

[11] Y. Ilamed, Bull. Res. Counc. Israel 5A (1956), 197.

[12] E. M. Loebl, Group theory and its applications, Vol. II (New York and London: Academic Press, 1971).

[13] J. D. Louck, Amer. J. Phys. 31 (1963), 378 and J. Math. Phys. 6(1965), 1786. N. Mukunda, J. Math. Phys. 8 (1967), 1069. J. D. Louck and H. Galbraith, Rev. Mod. Phys. 44 (1972), 540.

[14] J. D. Louck and L. C. Biedenharn, J. Math. Phys. 11 (1970), 2368.

[15] M. Moshinsky, J. Math. Phys. 4 (1963), 1128. 
[16] D M O'Brien, A. L. Carey and A. Cant, Ann. Inst. Henri Poincaré, Section A, Physique Théorique.

[17] S. Okubo, Rochester Report UR-608 (1977).

[18] M. K F. Wong, J. Math. Phys. 8 (1967), 1899.

\section{Department of Mathematical Physics}

The University of Adelaide

Adelaide

South Australia 5001 